\title{
The impact of an electronic clinical decision support for pulmonary embolism imaging on the efficiency of computed tomography pulmonary angiography utilisation in a resource-limited setting
}

\author{
C Murthy, ${ }^{1} \mathrm{MB}$ ChB; R Davis, ${ }^{1} \mathrm{MB}$ ChB, MMed (Rad D), FCRad (Diag) (SA); C F N Koegelenberg, ${ }^{2}$ MB ChB, MMed (Int), FCP (SA), \\ FRCP (UK), Cert Pulm (SA), PhD; E M Irusen, ${ }^{2}$ MB ChB, FCP (SA), PhD; R D Pitcher, ${ }^{1}$ MB ChB, FCRad (Diag) (SA)

\begin{abstract}
${ }^{1}$ Division of Radiodiagnosis, Department of Medical Imaging and Clinical Oncology, Faculty of Medicine and Health Sciences, Stellenbosch University and Tygerberg Hospital, Cape Town, South Africa

${ }^{2}$ Division of Pulmonology, Department of Medicine, Faculty of Medicine and Health Sciences, Stellenbosch University and Tygerberg Hospital, Cape Town, South Africa
\end{abstract}

Corresponding author: C Murthy (cmurthy05@gmail.com)

Background. Pulmonary embolism (PE) is associated with high morbidity and mortality. Effective intervention requires prompt diagnosis. Computed tomography pulmonary angiography (CTPA) is sensitive and specific for PE and is the investigation of choice. Inappropriate CTPA utilisation results in unnecessary high radiation exposure and is costly. State-of-the-art electronic radiology workflow can provide clinical decision support (CDS) for specialised imaging requests, but there has been limited work on the clinical impact of CDS in PE, particularly in resource-constrained environments.

Objective. To determine the impact of an electronic CDS for PE on the efficiency of CTPA utilisation in a resource-limited setting.

Methods. In preparation, a PE diagnostic algorithm was distributed to hospital clinicians, explaining the combined role of the validated modified Wells score and the quantitative D-dimer test in defining the pre-test probability of PE. Thereafter, an automated, electronic CDS was introduced for all CTPA requests. Total CTPA referrals and the proportion positive for PE were assessed for three study phases: (i) prediagnostic algorithm; (ii) post-algorithm, pre-CDS; and (iii) post-CDS.

Results. The proportion of CTPAs positive for PE after CDS implementation was almost double that prior to introduction of the diagnostic algorithm (phase 1 v. $3,17.4 \%$ v. $30.7 \%$; $p=0.036$ ), with a correspondingly significant decrease in the proportion of non-positive CTPAs (phases 1 v. 3, 82.6\% v. 69.3\%; $p=0.015$ ) During phases 2 and 3, no CTPAs were requested for patients with a modified Wells score of $\leq 4$ and a documented negative D-dimer, indicating adherence to the algorithm.

Conclusion. Implementing an electronic CDS for PE significantly increased the efficiency of CTPA utilisation and significantly decreased the proportion of inappropriate scans.

S Afr Med J 2016;106(1):62-64. DOI:10.7196/SAMJ.2016.v106i1.9886

Pulmonary embolism (PE) is a common and potentially fatal condition ${ }^{[1,2]}$ with an annual incidence of 29 - 69 cases per 100000 population $^{[3-5]}$ and a $15-30 \%$ mortality rate ${ }^{[6]}$ if untreated. It is the most common preventable cause of death in hospital patients, accounting for $10 \%$ of all hospital deaths. ${ }^{[7]} \mathrm{A} 5$-year retrospective autopsy study ${ }^{[8]}$ implicated undiagnosed PE in $10 \%$ of cases.

The nonspecific and highly variable presentation of PE makes the clinical diagnosis challenging. ${ }^{[4]}$ Only $25-30 \%$ of patients with PE-compatible symptoms have objective evidence of thromboembolism. ${ }^{[4,9]}$ Furthermore, PE symptoms may be masked by comorbidities. ${ }^{[10]}$

Recent advances in multidetector computed tomography (MDCT) have decreased scan times and increased diagnostic accuracy. CT pulmonary angiography (CTPA) can now be completed in seconds, with $83 \%$ and $96 \%$ sensitivity and specificity for PE, respectively. ${ }^{[1]}$ The accuracy of CTPA and its ability to provide alternative diagnoses, coupled with short scan times and widespread availability, have contributed to most clinicians having a low threshold for requesting the examination. ${ }^{[12]}$ However, this low threshold has resulted in overutilisation, without a commensurate increase in PE diagnosis. ${ }^{[2,12]}$ In a recent study only $10-15 \%$ of CTPAs performed in a large emergency unit were positive for PE. ${ }^{[12]}$
The safe and effective management of suspected PE, utilising a diagnostic algorithm that combines a validated clinical decision rule, the $\mathrm{D}$-dimer test and $\mathrm{CTPA}^{[10]}$ has been documented in various studies in the past decade. ${ }^{[13-15]}$ The combination of a low Wells score $(\leq 4)$ and a negative D-dimer test has been shown to have a negative predictive value for $\mathrm{PE}$ of $>99.5 \%$, allowing patients with low clinical probability of PE to be spared CTPA and anticoagulants, while all other patients undergo definitive CTPA work-up. ${ }^{[13-15]}$

Inappropriate utilisation of CTPA results in exposure to unnecessary high doses of ionising radiation and potential contrastrelated anaphylactic reactions and is costly, particularly in a resourcelimited setting. ${ }^{[10]}$

The technical advances in diagnostic imaging over the past four decades have been paralleled by major developments in information technology. Filmless and paperless digital radiology departments, utilising electronic workflow, are now commonplace in wellresourced healthcare environments. ${ }^{[16]}$ In addition, digital imaging and electronic workflow are increasingly being introduced into resource-limited settings, where they have particular benefit in supporting remote reporting via teleradiology. ${ }^{[17]}$ Electronic workflow in radiology is driven by the radiology information system (RIS), which has a number of efficiency-enhancing features. These include the capacity for clinicians to request imaging studies electronically 
and to be guided in real time by embedded, evidence-based imaging algorithms during the 'order entry' process. Decision-support systems for advanced imaging are being implemented with increased frequency, but evidence of their effectiveness in reducing inappropriate imaging utilisation is limited. This is particularly true for resource-limited healthcare environments, where improved efficiency and cost-saving are pivotal. ${ }^{[18]}$

We therefore aimed to determine the impact of an electronic clinical decision support (CDS) for PE on the efficient utilisation of CTPA in a resource-limited setting.

\section{Methods}

\section{Research site}

The study was conducted in a 1386 -bed public-sector tertiary-level teaching hospital in the Western Cape Province of South Africa. The hospital performs approximately 180000 radiological examinations annually and has a filmless, digital radiology department with an RIS-driven electronic workflow. Before December 2013, all imaging requests were submitted in hard copy, using standard request forms; thereafter all requests were generated electronically by way of the RIS. The study was approved by the institutional Health Research Ethics Committee and Hospital Management: Faculty of Medicine and Health Sciences of Stellenbosch University and Tygerberg Hospital, respectively.

\section{Study design}

The study was conducted in three phases.

Phase 1 (baseline observation). This was a retrospective analysis before December 2012, which served to secure a reasonable quantum of data to represent the baseline observation.

Phase 2 (preparatory - clinical guideline without prompting). In December 2012, by way of preparation, a PE diagnostic algorithm was distributed to all hospital clinicians, outlining the combined role of the validated modified Wells score (Table 1) and the quantitative D-dimer test in defining the pre-test probability of PE (Fig. 1).

\begin{tabular}{ll} 
Table 1. Modified Wells score & \\
\hline Previous PE or DVT & 1.5 \\
Heart rate $>100 \mathrm{bpm}$ & 1.5 \\
Surgery or immobilisation within 4 weeks & 1.5 \\
Haemoptysis & 1 \\
Active cancer & 1 \\
Clinical signs of DVT & 3 \\
Alternative diagnois less likely than PE & 3 \\
Clinical probability & \\
PE unlikely & $\leq 4$ \\
PE likely & $>4$ \\
DVT = deep-vein thrombosis; bpm = beats per minute.
\end{tabular}

Phase 3 (CDS). In December 2013, coinciding with the implementation of electronic requests for diagnostic imaging, a CDS for $\mathrm{PE}$ was introduced, which appeared in real time whenever a CTPA was requested on the RIS. Clinicians were prompted to enter the Wells score and the $\mathrm{D}$-dimer test result, thereby defining the pre-test probability of $\mathrm{PE}$ and hence the appropriateness of the CTPA request.

Results were stratified as positive or nonpositive for PE.

Inclusion and exclusion criteria. All requested CTPAs were approved by a radiology consultant prior to scanning. Consecutive CTPAs performed in the respective time periods were analysed, excluding patients who were pregnant, post partum or $<18$ years of age.

\section{CTPA technical and diagnostic parameters}

Examinations were performed on either a 6-slice or a 40-slice MDCT scanner, with transfer of axial acquisitions to a dedicated workstation (Intellispace Portal, Philips Healthcare, USA). CTPA technique and diagnostic criteria for PE were constant across all study phases.

Examinations were initially interpreted by registrars in training as radiologists. The final report of the duty consultant radiologist served as the reference standard.

\section{Statistical analysis}

Data were collected on a customised Microsoft Excel spreadsheet and analysed in Statistica 12. Continuous data were analysed using means and standard deviations (SDs), with $95 \%$ confidence intervals (CIs). The proportion of positive and non-positive examinations during each of the three phases was analysed using Pearson's $\chi^{2}$ test. In cases where small frequencies were observed, exact methods tested the nature of the association. A 5\% significance level $(p<0.05)$ was applied.

\section{Results \\ Patients}

A total of 603 CTPA examinations were performed across the three study phases; 424 patients (phase $1 n=149$, phase $2 n=174$, phase $3 n=101$ ) were included in the analysis and, 179 were excluded ( $<18$ years $n=3$, pregnant $n=56$, postpartum $n=115$, indications other than PE $n=5$ ) (Table 2). Patient demographics are presented in Table 3.

Shortness of breath was the most common symptom, present in 354 patients (83.5\%); other symptoms were chest pain $(n=130$, $30.7 \%)$, limb swelling $(n=53,12.5 \%)$ and haemoptysis $(n=27,6.4 \%)$.

The mean (SD) Wells score was 4.77 (1.5). During the prospective phases (2 and 3 ), no CTPAs were requested for patients with a modified Wells score $\leq 4$ and a negative $\mathrm{D}$-dimer test, indicating referring clinician adherence to the diagnostic guideline.

\section{CTPA findings}

The proportion of CTPAs positive for PE increased across the three phases (17.4\% v. $22.4 \%$ v. $31.7 \%$, respectively), with a corresponding decrease in the proportion of non-positive examinations over the same periods $(82.6 \% \mathrm{v}$. $77.6 \%$ v. 69.3\%, respectively) (Fig. 2).

Although the difference between phases 1 and $2(17.4 \%$ v. $22.4 \%$; $p=0.267)$ and between phases 2 and $3(22.4 \%$ v. $31.7 \% ; p=0.128)$ was not significant, the trend was clear and the overall difference between phases 1 and $3(17.4 \%$ v. $31.7 \%$; $p=0.014)$ was significant.

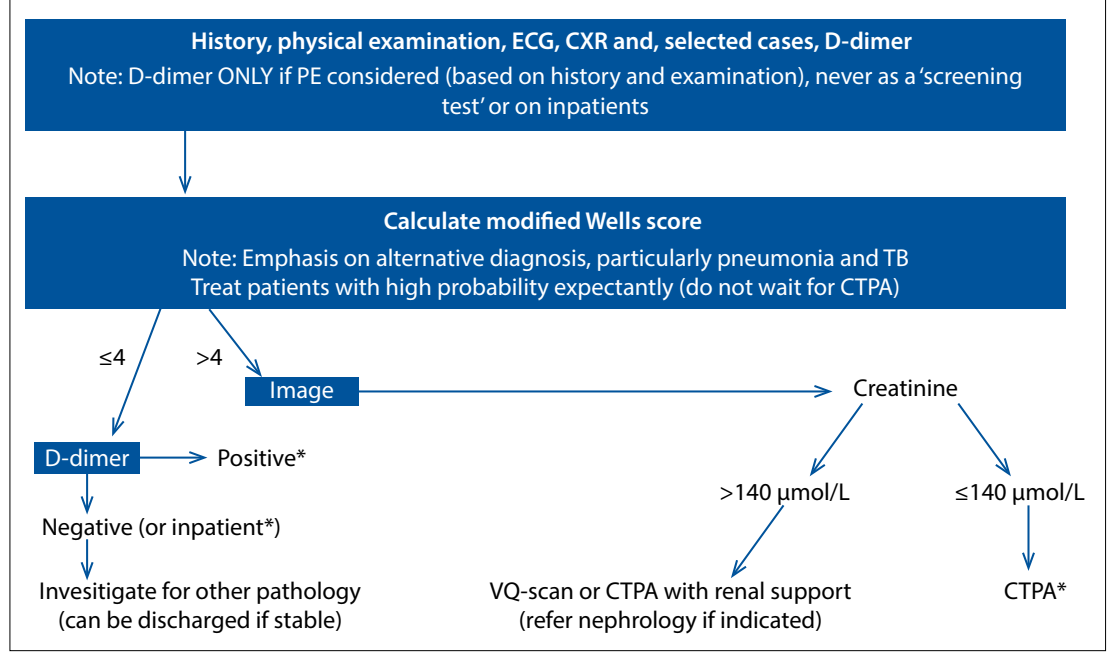

Fig. 1. Pulmonary embolism diagnostic guideline. ( $E C G=$ electrocardiograph; $C X R=$ chest $X$-ray; $T B=$ tuberculosis; VQ-scan = ventilation-perfusion scan; ${ }^{\star}$ Only image if no other explanation can be found.) 


\section{Discussion}

In this first study of its kind in a resourcelimited environment, we have shown that the phased implementation of a computerprompted pre-test probability scoring system for PE almost doubled the efficiency of CTPA and significantly decreased the number of inappropriate scans.

Both our $17.4 \%$ positive CTPA yield for PE in phase 1 and our $30.7 \%$ phase 3 yield are substantially higher than the pre-intervention (3 - 10\%) and post-intervention (10 - 17\%) ranges documented in recent similar studies ${ }^{[21-25]}$ in well-resourced environments. Furthermore, our overall $82 \%$ increased CTPA yield from baseline $(17.4 \%$ v. $30.7 \%)$ compares very favourably work of Prevedello et al. , $^{[22]}$ Drescher et al. ${ }^{[23]}$ and Raja et al. ${ }^{[24]}$ reported $37 \%$ (9.3 v. 12.6), $53 \%(8.3 \%$ v. $12.7 \%)$ and $69 \%(5.8 \%$ v. $9.8 \%)$ improvements after intervention, respectively. Dunne et al.s ${ }^{[21]}$ study of hospitalised patients yielded a modest $16.3 \%$ overall improvement (10.4\% v. 12.1\%). Soo Hoo et al. ${ }^{[11]}$ documented a striking $430 \%$ increased CTPA yield from a very low baseline $(3.1 \%$ v. $16.5 \%)$ in a general radiology department.

Our results are testimony to our clinicians' acceptance of, and adherence to, a simple $\mathrm{PE}$ diagnostic algorithm, reinforced by electronic CDS. Our experience differs from that documented by Drescher et al. ${ }^{[23]}$ who found that the CDS was poorly accepted by

\begin{tabular}{|c|c|c|c|}
\hline & $\begin{array}{l}\text { Positive, } \\
n(\%)\end{array}$ & $\begin{array}{l}\text { Non-positive, } \\
n(\%)\end{array}$ & Total \\
\hline Phase 1 & $26(17.4)$ & $123(82.6)$ & 149 \\
\hline Phase 2 & $39(22.4)$ & $135(77.6)$ & 174 \\
\hline Phase 3 & $31(30.7)$ & $70(69.3)$ & 101 \\
\hline Total & 96 & 328 & 424 \\
\hline
\end{tabular}
with recent studies. The emergency unit-based

emergency physicians, partly as a result of increased computer time, leading to selective use and decreased impact on overall yield, with ultimate removal of the PE from the computer order entry. Our substantial decrease in the proportion of inappropriate scans over time has considerable economic benefit, reflected in savings on consumables, and technician and radiologist time, ${ }^{[15]}$ enhancing overall institutional cost-effectiveness and efficiency. The success of the PE CDS bodes well for similar initiatives in a broad range of clinical settings, since numerous clinical algorithms are well suited to electronic prompting in radiology with a similar potential impact on clinical efficiency.

Strengths of our study were the two prospective study phases and the comprehensive availability of patient data on the RIS. A minor limitation was the lack of definitive diagnostic work-up, with either subtraction angiography or ventilationperfusion scanning, of the small proportion (6.3\%) of patients with an equivocal CTPA. Lastly, this study had limited power in distinguishing between the three phases and had limited external validity.

Since ours was a derivation cohort, we recommend validation of the results in a larger, prospective cohort. However, this intervention demands rigorous compliance and mindful application of the clinical decision rule. Hospital education programmes should include existing diagnostic guidelines, thereby encouraging its implementation by clinicians.

\section{Table 3. Patient demographics}

\section{Age (years), mean}

Gender, male/female ratio

Inpatient, $n(\%)$

Outpatient, $n(\%)$

$211(49.8)$

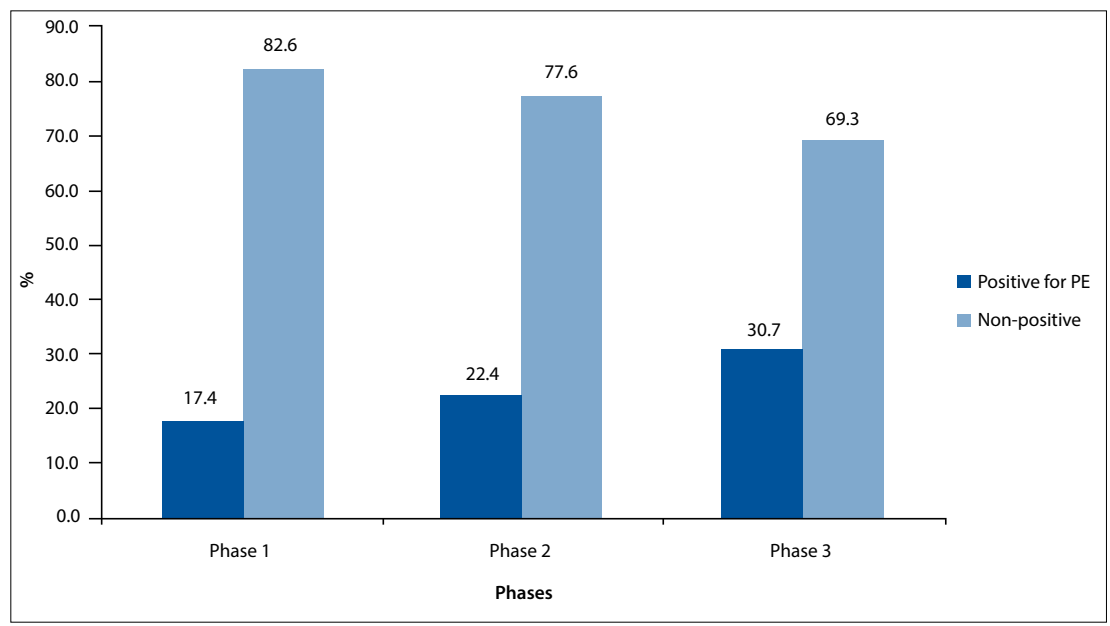

Fig. 2. Comparison of the proportion of positive and non-positive results for the three phases.
References

1. Soderberg M, Brohult J, Jorfeldt L, et al. The use of D-dimer testing and Wells score in patients with high probability for acute pulmonary embolism. J Eval Clin Pract 2009;15(1):129-133. [http:// dx.doi.org/10.1111/j.1365-2753.2008.00967.x]

2. Rohacek M, Buatsi J, Szucs-Farkas Z, et al. Ordering CT pulmonary angiography to exclude pulmonary embolism: Defense versus evidence in the emergency room. Intensive Care 2012;38(8):1345-1351. [http://dx.doi.org/10.1007/s00134-012-2595-z] 2012;38(8):1345-1351. hittp://dx.doi.org/10.1007/s00134-012-2595-2 3. Silverstein MD, Heit JA, Mohr DN, et al. Trends in the incidence of deep vein thrombosis and pulmonary embolism: A $25-y$ population-based study. Arch Intern Med 1998;158(6):585-593. 4. Lee AY, Hirch J. Diagnosis and treatment
thromboembolism. Annu Rev Med 2002:53(1):15-33.

thromboembolism. Annu Rev Med 2002;53(1):15-33.
5. Lucignani G, Pistolesi M. Diagnosing pulmonary embolism: Clinical problem or methodological issue? Eur J Nucl Med Mol Imaging 2009;36(3):522-528.[http://dx.doi.org/10.1007/s00259-008-1059-8]

6. Heit JA, Silverstein MD, Mohr DN, et al. The epidemiology of venous thromboembolism in the community. Thromb Haemost 2001;86(1):452-463.

7. Jacobson BF, Louw S, Mer M, et al. Venous thromboembolism: Prophylactic and therapeutic practice guideline. S Afr Med I 2013;103(4):260-267. [http://dx.doi.org/10.7196/SAMJ.6706]

8. Sandler DA, Martin JF. Autopsy proven pulmonary embolism in hospital patients: Are we detecting enough deep vein thrombosis? J Royal Soc Med 1989;82(4):203-205. [http://dx.doi. thrombosis? J Royal Soc Med 197
org/10.1177/014107688908200407]

9. The PIOPED Investigators. Value of the ventilation/perfusion scan in acute pulmonary embolism. Results of the Prospective scan in acute pulmonary embolism. Results of the Prospective
Investigation of Pulmonary Embolism Diagnosis (PIOPED). JAMA Investigation of Pulmonary

0. Wells PS, Anderson DR, Rodger M, et al. Derivation of a simple clinical model to categorize patients probability of pulmonary embolism: Increasing the models utility with the SimpliRED D-dimer. Thromb Haemost 2000;83(3):416-420.

11. Soo Hoo GW, Wu CC, Vazirani S, et al. Does a clinical decision rule using d-dimer level improve the yield of pulmonary CT angiography? AJR Am J Roentgenol 2011;196(5):1059-1064. [http:// dx.doi.org/10.2214/AJR.10.4200]

12. Shujaat A, Shapiro JM, Eden E. Utilization of CT pulmonary angiography in suspected pulmonary embolism in a major urban

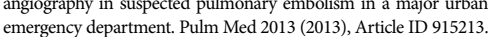
emergency department. Pulm Med 2013

13. Writing group for the Christopher Study Investigators. Effectivenes of managing suspected pulmonary embolism using an algorithm combining clinical probability, D-Dimer testing and computed tomography. JAMA 2006;295(2):172-179. [http://dx.doi. org/10.1001/jama.295.2.172]

14. Pasha SM, Klok FA, Snope JD, et al. Safety of excluding acute pulmonary embolism based on an unlikely clinical probability by the Wells rule and normal D-Dimer concentration: A meta-analysis. Thromb Res 2010;125(4):e123-e127. [http://dx.doi.org/10.1016/j. thromres.2009.11.009]

15. Ong CW, Malipatil V, Lavercombe M, et al. Implementation of a clinical prediction tool for pulmonary embolism diagnosis in a tertiary teaching hospital reduces the number of computed a tertiary teaching hospital reduces the number of computed tomography pulmonary angiograms performed. Intern Med
[http://dx.doi.org/10.1111/j.14455994.2012.02926.x]

16. Nance JW, Meenan C, Nagy P. The future of the radiology information system. AJR Am J Roentgenol 2013;200(5):1064-1070. [http://dx.doi.org/10.2214/ajr.12.10326]

7. Benjamin M, Aradi Y, Shreiber R. From shared data to sharing workflow: Merging PACS and teleradiology. Eur J Radiol 2010;73(1):3-9. [http://dx.doi.org/10.1016/j.ejrad.2009.10.014]

18. Blackmore CC, Mecklenburg RS, Kaplan GS. Effectiveness of clinical decision support in controlling inappropriate imaging J Am Coll Radiol 2011;8(1):19-25 [http://dx.doi.org/10.1016/j. jacr.2010.07.009]

19. Hedner C, Sundgren PC, Kelly AM. Associations between presence of relevant information in referrals to radiology and prevalence rates in patients with suspected pulmonary embolism. J Acad Radiol 2013;20(9):1115-1121. [http://dx.doi.org/10.1016/j acra.2013.05.010]

20. Mittadodla PS, Kumar S, Smith E, et al. CT pulmonary angiography: An over-utilized imaging modality in hospitalized patients with suspected pulmonary embolism. J Community Hosp Intern Me Perspect 2013;3(1). [http://dx.doi.org/10.3402/jchimp.v3i1.20240]

21. Dunne RM, Ip IK, Abbett S, et al. Effect of evidence-based clinical decision support on the use and yield of CT pulmonary angiographic imaging in hospitalized patients. Radiology 2015;276(1):167-174 [http://dx.doi.org/10.1148/radiol.15141208]

22. Prevedello LM, Raja AS, Ip IK, et al. Does clinical decision support reduce unwarranted variation in yield of $\mathrm{CT}$ pulmonary angiogram? Am J Med 2013;126(11):975-981. [http://dx.doi.org/10.1016/j. amjmed.2013.04.018]

23. Drescher FS, Chandrika S, Weir ID, et al. Effectiveness and acceptability of a computerized decision support system using modified Wells criteria for evaluation of suspected pulmonary embolism. Ann Emerg Med 2011;57(6):613-621. [http://dx.doi. embolism. Ann Emerg Med 2011;57/6.613
org/10.1016/j.annemergmed.2010.09.018]

24. Raja AS, Ip IK, Prevedello LM, et al. Effect of computerized clinical decision support on the use and yield of CT pulmonary angiography in the emergency department. Radiology 2012;262(2):468-474 [http://dx.doi.org/10.1148/radiol.11110951] 